\title{
Review of: BRCA1 and cyclin D1: gate keepers in hormone responsive tissues?
}

\author{
A. Griekspoor, T. C. Margarido, W. Zwart, R. Michalides \\ Department of Tumor Biology, The Netherlands Cancer Institute, Plesmanlaan, Amsterdam, The Netherlands.
}

\section{Citation of original article:}

C. Wang et al. Cancer Research 2005; 65(15): 6557-6567.

\begin{abstract}
of the original article:
The cyclin $D 1$ gene is frequently overexpressed in human breast cancer and is capable of inducing mammary tumorigenesis when overexpressed in transgenic mice. The BRCA1 breast tumor susceptibility gene product inhibits breast cancer cellular growth and the activity of several transcription factors. Herein, cyclin D1 antagonized BRCA1-mediated repression of estrogen receptor $\alpha(E R \alpha)$-dependent gene expression. Cyclin $D 1$ repression of BRCA1 function was mediated independently of its cyclin-dependent kinase, retinoblastoma protein, or p160 (SRC-1) functions in human breast and prostate cancer cells. In vitro, cyclin D1 competed with BRCA1 for $\mathrm{ER} \alpha$ binding. Cyclin D1 and BRCA1 were both capable of binding ER $\alpha$ in a common region of the ER $\alpha$ hinge domain. A novel domain of cyclin D1, predicted to form a helix-loop-helix structure, was required for binding to $\mathrm{ER} \alpha$ and for rescue of BRCA1-mediated $\mathrm{ER} \alpha$ transcriptional repression. In chromatin immunoprecipitation assays, $17 \beta$-estradiol $\left(E_{2}\right)$ enhanced $E R \alpha$ and cyclin D1 recruitment to an estrogen response element (ERE). Cyclin D1 expression enhanced ER $\alpha$ recruitment to an ERE. $E_{2}$ reduced BRCA1 recruitment and BRCA1 expression inhibited $\mathrm{E}_{2}$-induced $\mathrm{ER} \alpha$ recruitment at $12 \mathrm{~h}$. Cyclin $\mathrm{D} 1$ expression antagonized BRCA1 inhibition of ER $\alpha$ recruitment to an ERE, providing a mechanism by which cyclin D1 antagonizes BRCA1 function at an ERE. As cyclin D1 abundance is regulated by oncogenic and mitogenic signals, the antagonism of the BRCA1-mediated ER $\alpha$ repression by cyclin D1 may contribute to the selective induction of BRCA1-regulated target genes.
\end{abstract}

\section{Review}

In 1866, the French physician Paul Broca described for the first time the existence of breast cancer-prone families. It took until 1994 before the first BReast

Correspondence to: Rob Michalides, Department of Tumor Biology, The Netherlands Cancer Institute, Plesmanlaan, 121, 1066CX, Amsterdam, The Netherlands. E-mail: r.michalides@nki.nl; Tel: +31 20 5122022; Fax: +31 205122029

Received: 10/11/05

Accepted: 24/11/05

First published online 22/03/06

BCO/509/2005/JC
CAncer susceptibility gene (BRCA1) was cloned, soon followed by the identification of a second gene (BRCA2) [1]. Together, mutations in these two genes are responsible for nearly all of the $5 \%$ of breast cancers with a hereditary origin, although the quest for one or more BRCA genes still continues. Carriers of BRCA mutations not only show predisposition to breast cancer, but also to early onset of ovarian cancer and increased risk of developing other hormoneresponsive tumor types.

Both BRCA1 and 2 are directly involved in DNA repair and maintenance of genomic integrity [2]. Loss of these functions could explain predisposition to 
cancer, but not why mutation carriers are more prone to develop tumors in hormone responsive tissues. The discovery that BRCA1 interacts with the estrogen receptor (ER) and suppresses its transcriptional activity, gave new insights into this question [3]. This finding links BRCA1 directly to the central players in tumorigenesis of endocrine tissues.

Wang et al. now show that another key player, cyclin D1, competes with BRCA1 for the interaction with the ER [4]. The balance between the tumor suppressor BRCA1 and the oncogene cyclin D1 might be a decisive factor in driving tumor formation. BRCA1 loss or mutation leaves cyclin D1 activity unbalanced, leading to excessive ER activity.

The cyclin D1 gene encodes the regulatory subunit of a cyclin D1:cdk complex that phosphorylates and inactivates the retinoblastoma tumor suppressor, thereby driving the cell through the G1 phase of the cell cycle. Overexpression of cyclin D1 is found in $30-40 \%$ of breast cancers and is associated with poor prognosis of ER-positive breast cancer. Cyclin D1 also shows a cdk-independent activity in stabilizing the interaction between ER and SRC-1 via direct binding to both proteins [5]. Thereby, it enhances the estradiol-mediated activation of ER. The important role of cyclin D1 in breast tissue is exemplified by mouse studies, in which cyclin D1 knockout mice lack proper development of the mammary glands, while overexpression of cyclin D1 in a transgenic mouse model leads to the early onset of mammary carcinoma [6]. ER regulates the expression of the gene encoding cyclin D1, therefore the direct stimulation of the receptor by the cyclin D1 protein leads to an interesting positive feedback loop. This avalanche effect (activation of ER leads to cyclin D1, which stimulates ER to induce more cyclin D1 production) ensures a gradual overcoming of a threshold set by the inhibitory effect of BRCA1 on ER. Inactivation of cyclin D1 after transition through the $G_{1}$ phase of the cell cycle ends the role of cyclin D1.

A similar but negative feedback loop was found between BRCA1 and ER activity through the regulation of aromatase. In addition to direct suppression of ER activity, wt BRCA1 also inhibits aromatase expression, thereby lowering estrogen levels and further reducing ER-mediated transcription [7]. Indeed, BRCA1 mutation carriers have a higher breast density, indicative of excessive ER signaling.

Taken together, these examples illustrate the opposing effects of BRCA1 and cyclin D1 on ER as described by Wang et al. [4]. Their balance seems to be a decisive factor in the $G_{1} / S$ transition of hormonedependent cells. BRCA1 activity inhibits the positive feedback loop between cyclin D1 and ER, possibly resulting in a delay of the $G_{1} / S$ transition. It will be interesting to evaluate if this is related to the role of BRCA1 in the transcriptional response to DNA damage. During the $\mathrm{G}_{1} / \mathrm{S}$ phase transition, BRCA1 mRNA is upregulated and BRCA1 protein is hyperphosphorylated. Still, it remains to be tested how the binding between BRCA1 and ER is affected by phosphorylation and the presence of DNA damage.

Interestingly, while in the breast and ovary ER is inhibited by functional BRCA1, in prostate the androgen receptor (AR) is activated. However, cyclin D1 shows opposite effects as well, while it stimulates ER, it inhibits AR [8]. Thus, although the roles of both proteins seem to have switched, their balance also determines AR activity. This may explain why men carrying BRCA1 mutations are prone to develop prostate cancer.

Much of the scepticism about a direct role of the interaction between BRCA1 and ER in tumorigenesis, comes from the observation that the majority of breast tumors from mutation carriers are ER negative. Interestingly, these tumors are also cyclin D1 negative, altogether resulting in a basal phenotype [9]. Most sporadic tumors are ER positive and present a luminal phenotype, suggesting that sporadic and hereditary tumors develop along different pathways. Still, preventive removal of hormone producing organs or treatment with anti-estrogens significantly reduces the risk of tumors in BRCA1 mutation carriers. A possible explanation would be that the ER negative tumors represent a late stage of tumorigenesis in which ER expression is no longer needed and is therefore lost. Other researchers explain this observation by referring to stimulatory effects of surrounding ER positive cells on the stem cell compartment. Still, the question to what extend the loss of the BRCA1:ER interaction is causing the development of tumors in some or all BRCA1 mutation carriers remains. In this respect, it would be interesting to see whether there is any discrepancy between the types of mutations found in the $25 \%$ of BRCA 1 tumors that are still ER positive vs. those that show the basal phenotype. For instance, are BRCA1 point mutations found in the ER positive tumors indeed more clustered around the N-terminal ER interaction domain? Correlating mutations and truncations in the different BRCA1 protein domains with microarray based tumor subtyping may help to unravel the driving forces in tumorigenesis.

A greatly overlooked aspect of this discussion is a possible role for $\mathrm{ER} \beta$. In contrast to $\mathrm{ER} \alpha, \mathrm{ER} \beta$ is highly expressed in BRCA1 breast tumors, and could account for the observed responses to hormone [10]. ER $\beta$ is known to counteract ER $\alpha$-induced gene transcription. This can occur via heterodimerization, distinct regulation of promoters by ER $\beta$ and/or ER $\beta$ specific genes that are probably related to the antiproliferative properties of this receptor. Activation of 
ER $\beta$ by tamoxifen could thus explain the clinical benefits. Therefore, it is important to address whether the observations made by Wang et al. [4] are also valid for ER $\beta$. Does BRCA1 bind ER $\beta$ as well, and if so what is the effect on transcriptional activity of this receptor? The same questions should be asked for cyclin D1 in order to understand both sides of this important balance in hormone responsive tissues.

\section{References}

1. Narod S, Foulkes W. BRCA1 and BRCA2: 1994 and beyond. Nat Rev Cancer 2004; 4: 665-676.

2. Yoshida K, Miki Y. Role of BRCA1 and BRCA2 as regulators of DNA repair, transcription, and cell cycle in response to DNA damage. Cancer Sci 2004; 95: 866-871.

3. Fan S, Ma YX, Wang C, et al. Role of direct interaction in BRCA1 inhibition of estrogen receptor activity. Oncogene 2001; 20: 77-87.

4. Wang C, Fan S, Li Z, et al. Cyclin D1 Antagonizes BRCA1 repression of estrogen receptor activity. Cancer Res 2005; 65: 6557-6567.
5. Zwijsen RM, Wientjens E, Klompmaker R, et al. CDKIndependent activation of estrogen receptor by cyclin D1. Cell 1997; 88: 405-415.

6. Sicinski P, Weinberg RA. A specific role for cyclin D1 in mammary gland development. J Mammary Gland Biol Neoplasia 1997; 2: 335-342.

7. Hu Y, Ghosh S, Amleh A, et al. Modulation of aromatase expression by BRCA1: a possible link to tissue-specific tumor suppression. Oncogene 2005; 24: 8343-8348.

8. Burd CJ, Petre CE, Moghadam H, et al. Cyclin D1 binding to the androgen receptor $\mathrm{NH} 2$-terminal domain inhibits AF2 association and reveals dual roles for AR co-repression. Mol Endocrinol 2005; 19: 607-620.

9. Brenton JD, Carey LA, Ahmed AA, Caldas C. Molecular classification and molecular forecasting of breast cancer: ready for clinical application? J Clin Oncol 2005; 23: 7350-7360.

10. Daidone MG, Veneroni S, Cappelletti V, et al. Estrogen receptor-beta expression in hereditary breast cancer. J Clin Oncol 2002; 20: 3752-3753. 